\title{
A close relative of the nuclear, chromosomal high-mobility group protein HMG1 in yeast mitochondria
}

(Saccharomyces cerevisiae/DNA-binding protein/replication/transcription/HMG box)

\author{
John F. X. Diffley*† and Bruce Stillman* \\ *Cold Spring Harbor Laboratory, Cold Spring Harbor, NY 11724; and †Imperial Cancer Research Fund, Clare Hall Laboratories, Blanche Lane, South \\ Mimms, Potters Bar, Hertfordshire, EN6 3LD, United Kingdom \\ Communicated by James D. Watson, May 31, 1991 (received for review April 19, 1991)
}

\begin{abstract}
ABF2 (ARS-binding factor 2), a small, basic DNA-binding protein that binds specifically to the autonomously replicating sequence $A R S 1$, is located primarily in the mitochondria of the yeast Saccharomyces cerevisiae. The abundance of ABF2 and the phenotype of $a b 2^{-}$null mutants argue that this protein plays a key role in the structure, maintenance, and expression of the yeast mitochondrial genome. The predicted amino acid sequence of ABF2 is closely related to the high-mobility group proteins HMG1 and HMG2 from vertebrate cell nuclei and to several other DNA-binding proteins. Additionally, ABF2 and the other HMG-related proteins are related to a globular domain from the heat shock protein hsp70 family. ABF2 interacts with DNA both nonspecifically and in a specific manner within regulatory regions, suggesting a mechanism whereby it may aid in compacting the mitochondrial genome without interfering with expression.
\end{abstract}

It has been proposed that the contemporary mitochondrial genome evolved from an ancestral endosymbiont genome that has been pared down to include only the genes required for oxidative phosphorylation (reviewed in refs. 1 and 2). Replication and expression of the mitochondrial DNA (mtDNA) are controlled by nuclear genes, but the replication and segregation of mtDNA are independent of the corresponding events in the nucleus. Although advances have been made in understanding the general mechanism of transcription and replication of mtDNA, especially in animal cells (3), little is known about the role of nuclear proteins in controlling these events.

As part of a search for proteins that bind to nuclear origins of DNA replication in the yeast Saccharomyces cerevisiae, a small 20-kDa protein was identified that interacted with DNA in an unusual way $(4,5)$. This protein, ABF2 (ARS-binding factor 2), bound specifically to the autonomously replicating DNA sequence $A R S 1$, but also induced negative supercoiling of circular plasmid DNAs in the presence of a topoisomerase. We have further characterized this unusual DNA-binding protein. ABF2 contains two high-mobility group (HMG)-box DNA-binding motifs that have been found in a number of regulatory DNA-binding proteins. Interestingly, a substantial fraction of this HMG-like protein localizes to the mitochondria, where it is required for efficient maintenance of mtDNA and binds specifically to transcriptional and replication regulatory sequences.

\section{MATERIALS AND METHODS}

Strains. The parental $S$. cerevisiae strains used in this study were: SP1: MATa, ura3-52, his3, trp1-289, leu2-2,113, ade8, can1, ABF2 (6); W303-1: MATa/MATa, ade2-1/ade2-1, ura3-1/ura3-1, his3-11,15/his3-11,15, trp1-1/trp1-1, leu2$3,112 /$ leu2-3,112, canl-100/can1-100, ABF2/ABF2 (7);

The publication costs of this article were defrayed in part by page charge payment. This article must therefore be hereby marked "advertisement" in accordance with $18 \mathrm{U}$ U.S.C. $\$ 1734$ solely to indicate this fact.
YAM101 is W303-1 plus $A B F 2 / a b f 2:: U R A 3$ (this study), YAM102-YAM106 are haploid derivatives of YAM101. The EcoRI-Bgl II fragment from plasmid YRp7 containing the tryptophan-requiring gene $T R P I$ was used to replace the $B g l$ II-Sty I fragment from pAM1A (see Fig. 3A). This construct was digested with $E c o R I$ and used to transform the diploid strain W303-1 to tryptophan prototrophy (8). Transformants were analyzed by DNA blot hybridization to ensure that one copy of the $A B F 2$ gene was replaced with TRP1. The resulting strain, YAM101, was used for tetrad dissection (8) onto either yeast extract/peptone/dextrose (YPD containing glucose) or yeast extract/peptone/galactose (YPG) plates as described to generate strains YAM102-YAM106.

DNase 1 Cleavage-Inhibition Patterns (Footprinting) and Gel Shifts. Both methods have been described $(4,5)$.

Immunofluorescence and DNA Staining. Logarithmic-phase cells $\left(10^{8}\right)$ were fixed and processed for immunofluorescence as described (8). Total cell DNA was visualized with the DNA-specific dye $4^{\prime}, 6$-diamidino-2-phenylindole (DAPI). The antibody used to visualize ABF2 was a polyclonal antiserum from rabbit raised against purified ABF2 protein. Fluorescein isothiocyanate-conjugated goat anti-rabbit immunoglobulin was used as the second antibody.

Cloning and Sequencing. The following sequence from the amino terminus of purified ABF2 was determined by $D$. Marshak using an Applied Biosystems 475 protein sequencer: Lys-Ala-Ser-Lys-Arg-Thr-GIn-Leu-Arg-Asn-Glu-Leu-IleLys-GIn-Gly-Pro-Lys-Arg-Pro-Thr-Ser-Ala-His-Phe-LeuTyr-Leu-Gln-Asp. The underlined protein sequences were used to design the following two degenerate oligonucleotides: RTCYTGYARRTAYARRAARTG and AARGCWTCWAARMGRAC, where $\mathbf{R}=\mathbf{A}$ or $\mathbf{G}, \mathbf{Y}=\mathrm{T}$ or $\mathrm{C}, \mathbf{W}=\mathbf{A}$ or $\mathrm{T}$, and $\mathbf{M}=\mathbf{A}$ or $\mathbf{C}$. One hundred picomoles of each oligonucleotide was used to amplify the amino terminus-encoding region of the $A B F 2$ gene from 2 to $15 \mathrm{ng}$ of purified genomic DNA from the yeast strain SP1. Amplification was for 25 cycles in a Perkin-Elmer thermal cycler: $1.5 \mathrm{~min}$ at $94^{\circ} \mathrm{C}, 5$ min at $45^{\circ} \mathrm{C}$, and $3 \mathrm{~min}$ at $65^{\circ} \mathrm{C}$. The expected 89-base-pair (bp) fragment was obtained and excised from a preparative polyacrylamide gel run in Tris borate/EDTA buffer. Labeled probe was obtained by amplifying this 89-bp product with the same oligonucleotides for 10 cycles under the same conditions except that $\left[\alpha^{-32} \mathrm{P}\right] \mathrm{dTTP}$ was included at a final concentration of $1.25 \mu \mathrm{M}$ and unlabeled dTTP was omitted. This DNA was used to probe a partial HindIII library of genomic DNA from the yeast strain SP1 (6) cloned in the HindIII sites of the yeast vector YEp213. In this library (a gift from S. Cameron and M. Wigler, Cold Spring Harbor Laboratory), the

Abbreviations: DAPI, 4',6-diamidino-2-phenylindole; ARS, autonomously replicating sequence; HMG, high-mobility group; YPD, yeast extract/peptone/dextrose; YPG, yeast extract/peptone/ galactose; ABF2, ARS-binding factor 2; UBF, upstream binding factor; SRY, sex-determining region of Y chromosome; NHP6, nonhistone chromosomal protein 6; BiP, rat immunoglobulin heavy chain binding protein. 
average size insert is 6 kilobases $(\mathrm{kb})$. Colonies $\left(4.5 \times 10^{4}\right)$ were screened (approximately three genome equivalents) by standard methods, and a single strong positive colony was analyzed further. This plasmid (pAM1) contains an 11.5-kb HindIII fragment insert. A $1.6-\mathrm{kb} E c o R I$ fragment that hybridized to the labeled, amplified probe was subcloned into pUC118 (pAM1A). Deletions were generated by the method of Henikoff (9), and the sequence was determined by the method of Sanger et al. (10). $\ddagger$ [See Fig. $3 A$ for the sequence of the $A B F 2$ coding sequence and some of the $5^{\prime}$ and $3^{\prime}$ sequences. Note that the protein sequence originally determined contains one discrepancy with the predicted sequence (amino acid 50 is tyrosine in Fig. $3 A$ but histidine in the originally determined protein sequence cited above). A greater amount of purified ABF2 was subsequently resequenced and shown to contain a tyrosine residue at amino acid 50.]

Subcellular Fractionation. Subcellular fractionation of late logarithmic-phase yeast cells from 4 liters of YPG medium was performed as described (11). Percoll gradient-purified nuclei were obtained as described (8). Equal cell equivalents of each fraction were resolved on SDS $/ 17 \%$ polyacrylamide gels, electroblotted, and probed with a 1:250 dilution of anti-ABF2 or a 1:2500 dilution of antiserum to p32, an integral, mitochondrial membrane protein (11), a gift from $D$. Pain and G. Blobel (Rockefeller University). Antibody was visualized by probing blots with ${ }^{125}$ I-labeled donkey antirabbit IgG and subsequent autoradiography.

\section{RESULTS AND DISCUSSION}

ABF2 was initially identified in crude yeast extracts because of its unusual interactions with a yeast origin of nuclear DNA replication, $A R S 1(4,5)$. ABF2 has been purified extensively from whole-cell yeast extracts as a polypeptide of $20 \mathrm{kDa}$, and a polyclonal antiserum to the purified protein has been raised. Indirect immunofluorescence (Fig. 1B) revealed a punctate, cytoplasmic staining similar to the distribution of mtDNA (cytoplasmic DAPI staining; Fig. 1A). Subcellular fractionation (Fig. $1 C$ ) indicated that, like a known mitochondrial protein (p32), ABF2 was found in purified mitochondria, but a substantial amount of ABF2 was also found in the nucleus. Thus, we conclude that ABF2 is a mitochondrial protein and probably also a nuclear protein. The nuclear ABF2 protein that was detected by immunoblotting was not observed by immunofluorescence, perhaps because the antigen was masked under these conditions. Quantitative immunoblots and DNA-binding assays with crude extracts indicate that $A B F 2$ is an abundant protein, present at approximately 250,000 copies per cell (data not shown), or one molecule of ABF2 for every $15 \mathrm{bp}$ of mtDNA.

ABF2 exhibited several interesting DNA-binding properties. First, in cooperation with topoisomerase I, ABF2 induced the negative supercoiling of relaxed, closed-circular plasmids (ref. 5 and unpublished data), suggesting that ABF2 bound DNA by wrapping, like the prokaryotic $\mathrm{HU}$ protein (12) and the eukaryotic nucleosome (13). Second, while it bound many DNA sequences with similar affinity, it exhibited specific, phased binding to certain key regulatory sequences. Examples of this phased binding as visualized by DNase 1 footprinting are shown in Fig. 2. At the nuclear origin of replication, $A R S 1, \mathrm{ABF} 2$ bound at multiple, discrete sites across domains implicated as being important for $A R S$ function (Fig. 2A). Footprints on other ARSs (data not shown) indicate that phased binding is a general feature of ABF2 binding to chromosomal replication origins, whereas footprinting studies on other DNAs (e.g., pBR322) reveal generalized but not phased DNA binding.

¥The sequence reported in this paper has been deposited in the GenBank data base (accession no. M73753).
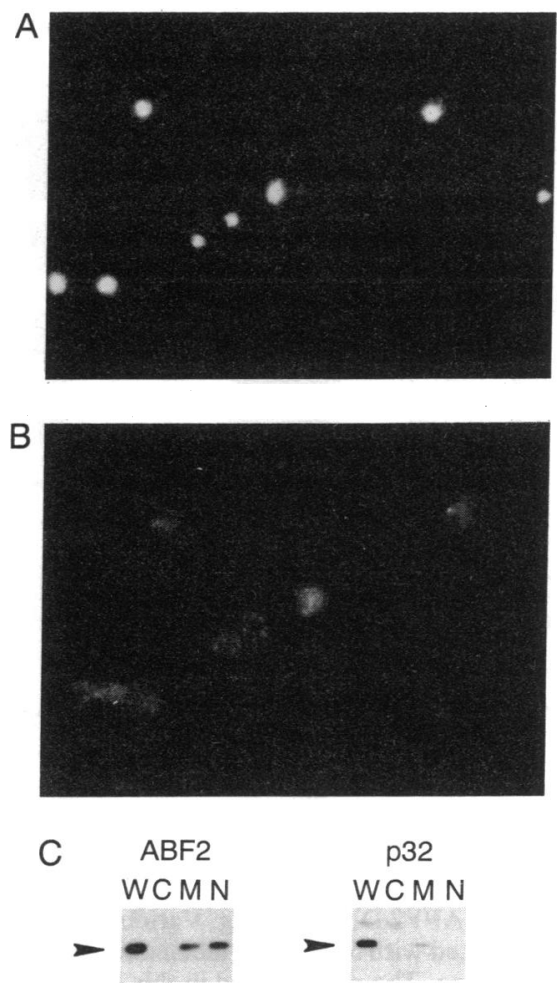

FIG. 1. Localization of ABF2 to the mitochondria. (A) DNA staining of nuclei and mitochondria in a wild-type strain with the dye DAPI. $(B)$ Localization of ABF2 to mitochondria by immunofluorescence with anti-ABF2 antisera. $(C)$ Subcellular localization of ABF2 protein determined by immunoblotting. Extracts from whole cells (lanes W), cytosol (lanes C), mitochondria (lanes M), and nuclei (lanes N) were prepared, and proteins were separated by gel electrophoresis. The $A B F 2$ protein (Left) or the p32 mitochondrial protein (Right) was detected by immunoblotting and indicated by arrows.

The mitochondrial localization of ABF2 together with its phased binding at nuclear replication origins suggested that binding of $\mathrm{ABF} 2$ at mitochondrial regulatory regions may also be phased. $R E P 2$ is a region from the yeast mitochondrial genome that contains a promoter for the mitochondrial RNA polymerase and has been inferred to contain one of several origins of mitochondrial DNA replication based on the phenomenon of hypersuppressivity (15). In addition to regions of general, nonspecific binding, $\mathrm{ABF} 2$ binds strongly to a single site immediately adjacent to the promoter where the mitochondrial RNA polymerase has previously been shown to bind specifically (16) (Fig. $2 B$ ).

Reiterations of a very short sequence containing only $A$ and $T$ residues derived from a region downstream from the RNA polymerase promoter at $R E P 2$ are still capable of replicating their genomes (17), suggesting that this region may contain a minimal origin of mtDNA replication. ABF2 binding to one of these repeated sequences exhibited phasing similar to that seen at the nuclear origin of replication, $A R S I$ (Fig. $2 C$ ). It is interesting that $R E P 2$ or these sequences derived from $R E P 2$ can also function as origins of DNA replication in the nucleus (14), suggesting that there may be some common feature(s) to mitochondrial and nuclear replication origins that is recognized by ABF2.

To understand further both the unusual DNA-binding activities of $A B F 2$ and the in vivo role of this protein, we have cloned the $A B F 2$ gene. Amino acid sequence was obtained from the amino terminus of the purified ABF2 protein and used to design two degenerate oligonucleotides that were used to amplify the corresponding region of the $A B F 2$ gene by the polymerase chain reaction from yeast genomic DNA. 
A
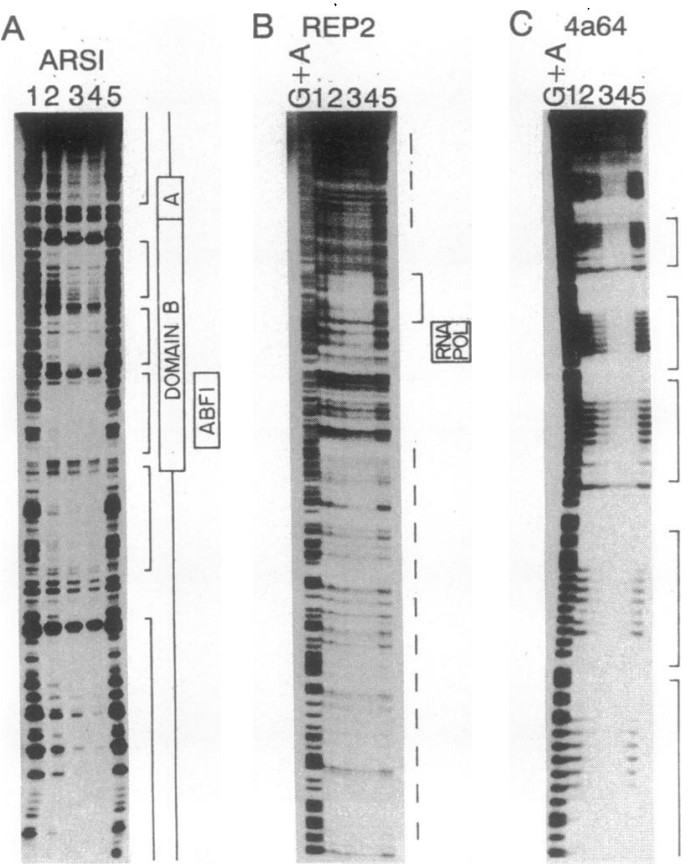

FIG. 2. Phased ABF2 DNA binding. Various amounts of purified ABF2 were incubated with $5^{\prime}-32$-end-labeled DNA and subjected to DNase 1 footprinting. The probes used in this experiment were: the HindIII-EcoRI fragment from pARS1.2 (4) containing nucleotides 616-927 of ARS1 and labeled at the HindIII site (A), the BamHIEcoRI fragment from the plasmid pGEMHS40N7 containing REP2 on a 760-bp Nde I fragment cloned into the Sma I site of pGEM32f(-) and labeled at the BamHI site (B), and the BamHI (partial digest) to Sal I fragment from the plasmid p[64] $]_{4}(14)$ labeled at the BamHI site (C). Lanes: $\mathbf{G}+\mathbf{A}$, a $\mathbf{G}+\mathbf{A}$-specific sequence cleavage (in $B$ and $C$ only); 1-5, 1 and 5, controls ( $\mathrm{ng}$ of ABF2); $2,30 \mathrm{ng}$ of ABF2; 3, 60 ng of $A B F 2 ; 4,90 \mathrm{ng}$ of $A B F 2$. In each case, the phased ABF2 binding sites are indicated by brackets, while general regions of unphased protection are indicated by dotted lines. Functional elements within these DNA sequences are shown to the right of each panel. Domains A and B of ARS1 have been described, as has the binding of ABF1 to domain $B$ of this sequence (4).

This amplified DNA was used as a probe to isolate a clone containing the $A B F 2$ gene from a yeast genomic library. The nucleotide sequence of the $A B F 2$ gene was determined and is shown in Fig. 3A. The amino-terminal amino acid sequence determined from the purified protein is underlined in the predicted amino acid sequence of the $\mathrm{ABF} 2$ protein. This sequence starts 26 amino acids downstream from the nearest potential start codon and these first 26 amino acids provide a good match to a mitochondrial signal sequence (1), consistent with the observed intracellular localization of ABF2. We note that, on the basis of the predicted amino acid composition, abundance, and ability to supercoil DNA, ABF2 resembles the previously characterized HM protein from yeast mitochondria (19).

The ABF2 amino acid sequence is closely related to the vertebrate, nonhistone chromosomal high-mobility group protein HMG1 (ref. 20; 23\% identical, 38\% conserved relative to the ABF2 protein). These HMG proteins consist of three domains; amino-terminal and central domains, which are closely related to each other; and an acidic carboxyl-terminal domain (21). ABF2 is composed of two domains closely related to the amino-terminal and central domains of HMG1 and HMG2 (hereafter referred to as HMG boxes), but does not contain a domain related to the carboxyl-terminal acidic domain of the HMG proteins (Fig. $3 B$ and $C$ ). Several other proteins also contain HMG boxes including the human RNA polymerase I transcription factor UBF (upstream binding factor; ref. 22), the product of the Schizosaccharomyces pombe mating type gene $M A T I-M_{c}(23)$, the $S$. cerevisiae nonhistone chromosomal protein NHP6 (24), and the product of the recently proposed candidate for the human testisdetermining gene $S R Y$ (sex-determining region of $Y$ chromosome; ref. 25). ABF2 (refs. 4 and 5 and Fig. 2), UBF (26), and HMG1 and HMG2 (27) have been shown to bind DNA while the others are likely to bind DNA because NHP6 is found to be associated with chromatin (28) and MAT1-M $\mathrm{M}_{\mathrm{c}}$ and SRY are key regulatory proteins $(23,25)$. Since the HMG boxes constitute virtually the entire $\mathrm{ABF} 2$ protein (Fig. $3 C$ ) and deletion analysis of UBF argues that the HMG box is involved in DNA binding (22), the HMG box appeared to be used as a DNA-binding motif by these different proteins. It is interesting to note that alignment of the many phased ABF2 binding sites (Fig. 2 and data not shown) or the UBF binding sites (29) does not reveal any easily recognizable consensus sequences, suggesting that some feature of DNA structure other than primary nucleotide sequence may be critical for the binding of HMG box proteins.

We also note that the HMG box exhibits weaker but nonetheless striking similarity to an independent globular domain found in the ATPase region of the heat shock protein hsp70 family (30), suggesting that the HMG box may be derived from an ancient globular domain.

One copy of the $A B F 2$ gene was replaced with the TRPI gene in a diploid yeast strain (W303-1), and replacement was verified by DNA blot hybridization. After sporulation of this strain, tetrads were dissected, plated onto complete medium containing dextrose (glucose), and analyzed. In most cases, all four spores were viable, generating two $\mathrm{TRP}^{+}$and two trp $^{-}$colonies. The four strains derived from one such tetrad were analyzed further. Protein extracts were made from each of the four strains and assayed for ARS1-specific DNAbinding activity. Fig. $4 A$ demonstrates that $A B F 2$ DNAbinding activity was missing from the two abf2- mutant strains and present in the two $A B F 2^{+}$wild-type strains. Furthermore, immunoblots on these same extracts probed with antibody raised against purified $A B F 2$ failed to detect any $20-\mathrm{kDa}$ protein in the two $a b f 2^{-}$mutant strains, but recognized a 20-kDa protein that comigrated in an SDS/ polyacrylamide gel with purified $A B F 2$ in the two $A B F 2^{+}$ strains (Fig. 4A). Finally, the null mutant strains lost all mitochondrial immunofluorescence with anti-ABF2 antibodies (data not shown). Therefore, the gene that we have isolated encodes the major, if not only, form of ABF2.

While $a b f 2^{-}$null mutants are viable, they exhibit a phenotype consistent with the mitochondrial localization of ABF2. In medium containing a fermentable carbon source like glucose, where mitochondrial genome function is dispensable, $a b f^{-}$null mutants grew nearly as well as wild type (Fig. $4 C$ ). In contrast, in media containing a nonfermentable carbon source like glycerol, where mitochondrial genome function is essential, this $a b f 2^{-}$mutant was unable to grow at all (Fig. 4C). This is not surprising, since this particular isolate has lost most or all mtDNA as determined by staining of whole cells with the DNA-specific dye, DAPI (data not shown).

The loss of mtDNA occurs rapidly in abf2- strains grown in glucose but was not the inevitable consequence of loss of ABF2. When tetrads from the diploid heterozygous for the $a b 2^{-}$null mutant were dissected directly onto plates containing glycerol as the sole carbon source, $a b 2^{-}$strains could grow and could be maintained indefinitely. Under these conditions, however, $a b f 2^{-}$strains exhibited a growth defect on medium containing glycerol, where mitochondrial gene expression was required (Fig. 4D), even though almost all of the cells appeared to contain wild-type levels of mtDNA based upon DAPI staining (data not shown). Thus, ABF2 appeared to be important for both the maintenance and the expression of the mitochondrial genome. 
A

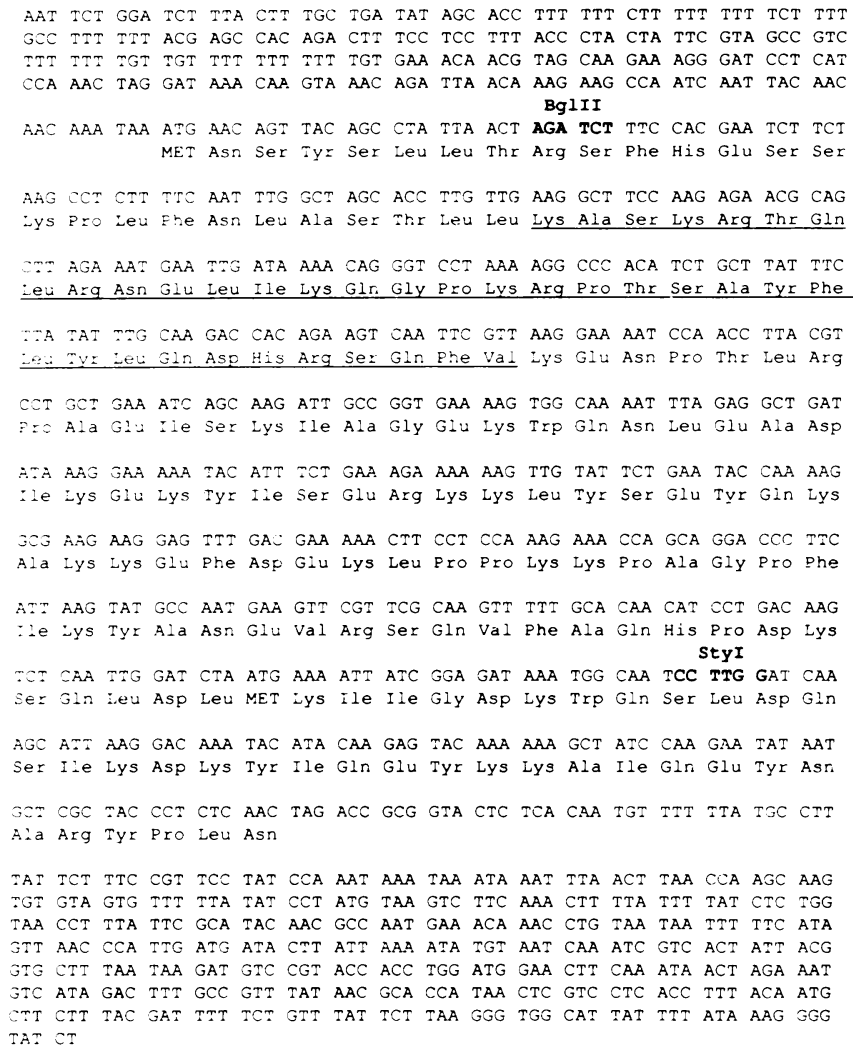

B

\section{ABF2 N-Term. HMG1 N-Term. UBF Box 1 UBF Box 2 ABF2 C-Term. HMG1 C-Term. UBF BOX 3 MAT1-M C-Term. NHP6 SRY

C

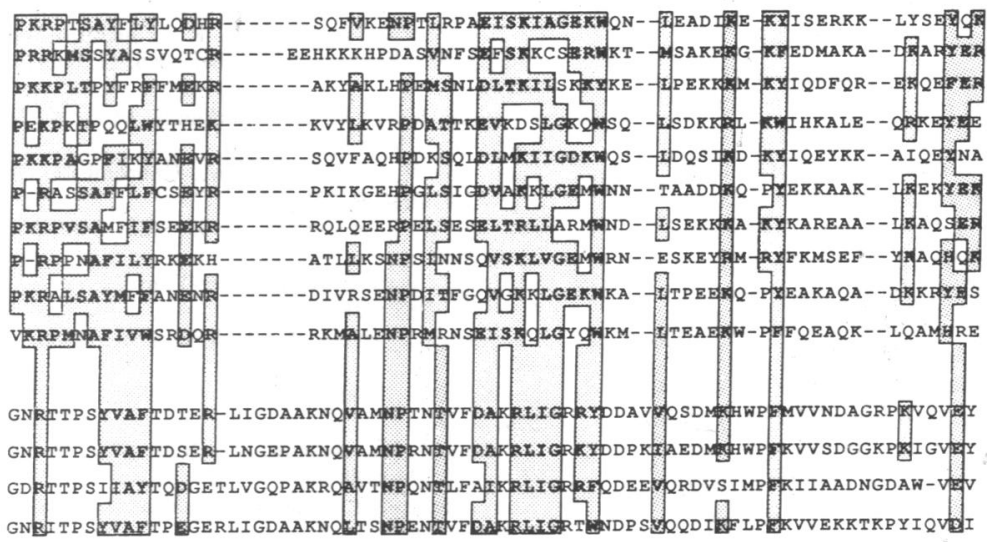

ABF2

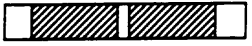

HMG1

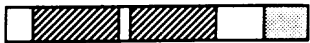

UBF

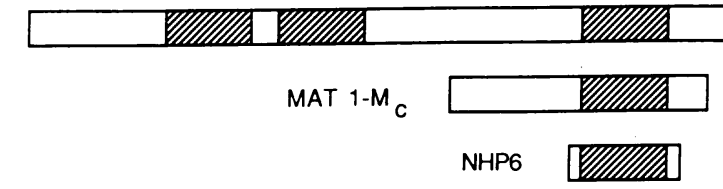

We suggest that the general ability of $A B F 2$ to bind nonspecifically to DNA by wrapping and its specific, phased binding at key regulatory sequences allow it to compact the yeast mitochondrial genome without interfering with efficient gene expression and replication. The binding of ABF2 adjacent to the RNA polymerase promoter (Fig. 2) and the defective growth of $a b f 2^{-}$null mutants on nonfermentable carbon sources (Fig. $4 C$ and $D$ ) suggest that ABF2 may also have some positive role in gene expression or replication.
FIG. 3. Cloning and sequencing of ABF2. (A) Sequence of the $A B F 2$ gene. $(B)$ Alignment of HMG boxes in several related proteins. $(C)$ Location of HMG boxes within these proteins. Searches of data banks were by the method of Pearson and Lipman (18), from which the similarities between ABF2, HMG1, HMG2, MAT1-M and rat immunoglobulin heavy chain binding protein (BiP) were determined. Similarities between ABF2 and UBF and between SRY and NHP6 were found subsequently upon publication of these sequences, and the other hsp70-related proteins were aligned based on their homology to BiP. Groups of amino acids considered to be conserved are: Ala-IleLeu-Met-Val, Tyr-Phe-Trp-His, ArgLys, Glu-Asp, Asn-GIn, and Ser-Thr. The HMG boxes in $C$ are indicated by hatched boxes, and acidic domains are indicated by stippled boxes.
The rapid loss of mtDNA would be consistent with this, since the maintenance of mtDNA requires mitochondrial gene expression (31). Recently, Parisi and Clayton (32) demonstrated that the human mitochondrial transcription factor 1 (mtTF1) is a 24-kDa protein containing two HMG boxes. mtTF1 binds to mtDNA promoters and activates transcription in vitro. It is therefore probable that $\mathrm{ABF} 2$ is the $S$. cerevisiae mtTF1 protein.

The mitochondrial genotype in yeast can influence nuclear 

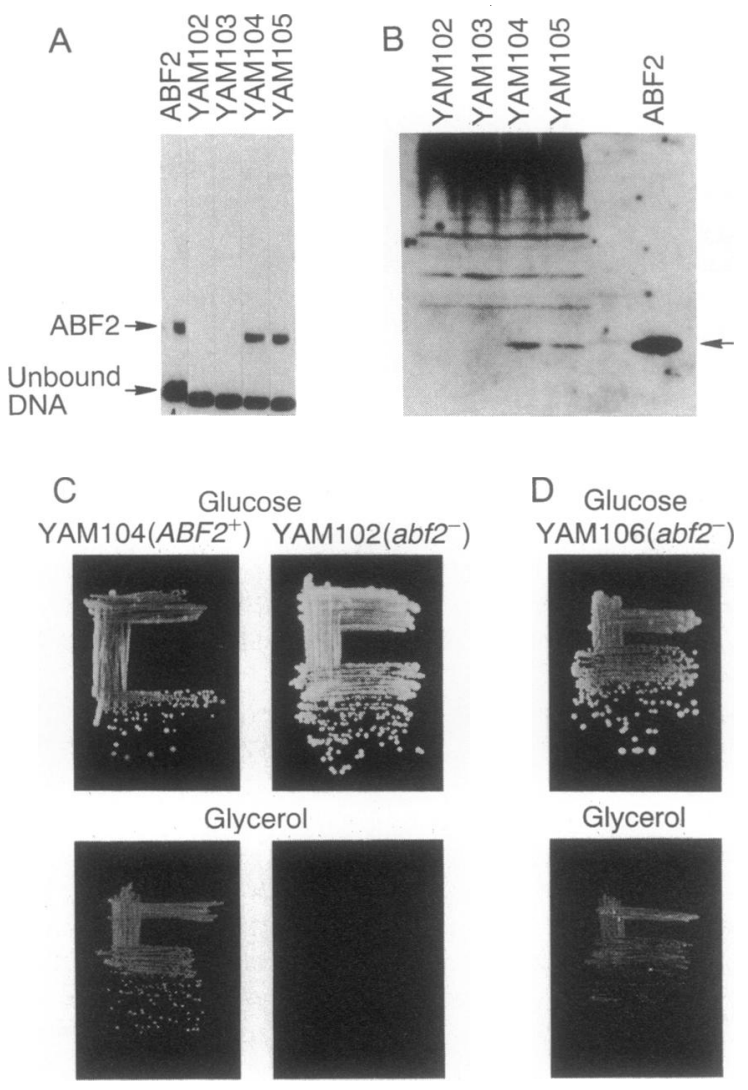

FIg. 4. Characterization of $a b f 2^{-}$strains. (A) ABF2 DNAbinding activity in mutant and wild-type strains. (B) ABF2 protein in mutant and wild-type strains. (C) Generation of $\rho^{0} a b f 2^{-}$strains. (D) The ability of abf $\rho^{+}$mutants to utilize nonfermentable carbon sources. Strain YAM101 was sporulated, and tetrads were dissected onto YPD (glucose) plates. After restreaking the colonies from one such tetrad to ensure purity, these strains were tested for their ability to grow in the absence of exogenous tryptophan. The strains YAM102 and YAM103 were $T R P^{+}$, while YAM104 and YAM105 were $t r p^{-}$. Extracts from these four strains were made by mixing late logarithmic-phase cells in buffer X (4) with glass beads. Each extract $(3 \mu \mathrm{g})$ was assayed for ABF2 DNA-binding activity by using the $A R S I$ sequences on the EcoRI-HindIII fragment from pARS1.4.1 as a probe (4). Purified ABF2 $(0.2 \mu \mathrm{g})$ was used as a control in this experiment $(A)$. These same extracts were probed for $A B F 2$ protein by using the anti-ABF2 polyclonal antiserum. Each extract (150 $\mu \mathrm{g})$ was subjected to electrophoresis in SDS/15\% polyacrylamide gels, electroblotted to nitrocellulose, and probed with anti-ABF2 antibodies ( ${ }^{125}$ I-labeled protein A was used to visualize antibody binding) $(B)$. These strains were tested for their ability to grow on glucose (YPD plates containing $15 \%$ glucose) or glycerol (YPG plates). Shown for simplicity are YAM102 $\left(a b 2^{-}\right)$and YAM104 $\left(\mathrm{ABF2}^{+}\right)$ $(C)$. The diploid YAM101 was sporulated again in rich sporulation medium, and tetrads were dissected directly onto YPG plates. All four spores routinely germinate yielding two large colonies that are trp ${ }^{-}$and two small colonies which are $T R P^{+}$. The ability of one of these small $T R P^{+}$strains, designated YAM106, to grow on glucose and glycerol is shown in $D$. Note the much smaller size of colonies on YPG of strain YAM106 relative to strain YAM104 shown in $C$.

gene expression (33), and the existence of shared transcription factors between the nucleus and mitochondria has been proposed (34). The localization of a portion of ABF2 to the nucleus suggests that it may play a role in coordinating nuclear-mitochondrial interactions, perhaps directly monitoring the amount of mtDNA. If, on the other hand, ABF2 has nuclear functions other than those related to mitochondrial function, they must either be nonessential or duplicated by some other gene product.
Because ABF2 is so closely related to the vertebrate HMG1 and HMG2 proteins, it is tempting to speculate that these proteins may have a related role in the nucleus, binding at regulatory regions, perhaps positioning nucleosomes with their acidic tail. For example, the region of $A R S 1$ exhibiting phased ABF2 binding (Fig. 2) is devoid of nucleosomes in situ and is bordered on at least one side by a precisely positioned nucleosome $(36,37)$. Furthermore, the regular pattern of nuclease sensitivity across this region is similar to that seen with ABF2 (Fig. 2A), suggesting that ABF2 or an ABF2-like protein may be responsible for this pattern in vivo.

We thank Naama Kessler for excellent technical assistance, Dan Marshak and Georgia Binns for performing the N-terminal amino acid sequence analysis, Walt Fangman for the REP plasmids and for discussions, Debkumar Pain and Gunter Blobel for the anti-p32 antiserum, and Barbara Weinkauff for typing the manuscript. This research was supported by a research grant from the National Institutes of Health (AI20460).

1. Attardi, G. \& Schatz, G. (1988) Annu. Rev. Cell Biol. 4, 289-333.

2. Gray, M. W. (1989) Annu. Rev. Cell Biol. 5, 25-30.

3. Clayton, D. A. (1991) Trends Biochem. Sci. 16, 107-111.

4. Diffley, J. F. X. \& Stillman, B. (1988) Proc. Natl. Acad. Sci. USA 85, 2120-2124.

5. Diffley, J. F. X. \& Stillman, B. (1988) Cancer Cells 6, 235-243.

6. Kataoka, T., Powers, S., McGill, C., Fassano, O., Strathern, J., Broach, J. \& Wigler, M. (1984) Cell 37, 437-445.

7. Rothstein, R. (1983) Methods Enzymol. 101, 202-211.

8. Rose, M. D., Winston, F. \& Hieter, P. (1989) Methods in Yeast Genetics (Cold Spring Harbor Lab., Cold Spring Harbor, NY).

9. Henikoff, S. (1987) Methods Enzymol. 155, 156-165.

10. Sanger, F., Nicklen, S. \& Coulson, A. R. (1977) Proc. Natl. Acad. Sci. USA 74, 5463-5467.

11. Pain, D., Murakami, H. \& Blobel, G. (1990) Nature (London) 347, 444-449.

12. Rouviere-Yaniv, J. \& Yaniv, M. (1979) Cell 17, $265-274$.

13. van Holde, K. E. (1989) Chromatin (Springer, New York).

14. Zweifel, S. G. \& Fangman, W. L. (1990) Yeast 6, 179-186.

15. de Zamaroczy, M., Marotta, R., Faugeron-Fonty, G., Goursot, R., Mangin, M., Baldacci, G. \& Bernardi, G. (1981) Nature (London) 292, 75-78.

16. Schinkel, A. H., Groot Koerkamp, M. J. A. \& Tabak, H. F. (1988) EMBO J. 7, 3255-3262.

17. Fangman, W. L., Henly, J. W., Churchill, G. \& Brewer, B. J. (1989) Mol. Cell. Biol. 9, 1917-1921.

18. Pearson, W. R. \& Lipman, D. J. (1988) Proc. Natl. Acad. Sci. USA 85, 2444-2448.

19. Caron, F., Jacq, C. \& Rouviere-Yaniv, J. (1979) Proc. Natl. Acad. Sci. USA 76, 4265-4269.

20. Paonessa, G., Frank, R. \& Cortese, R. (1988) Nucleic Acids Res. 15, 9077.

21. Reeck, G. R., Isackson, P. J. \& Teller, D. C. (1982) Nature (London) $300,76-78$.

22. Jantzen, H.-M., Admon, A., Bell, S. P. \& Tjian, R. (1990) Nature (London) 344, 830-836.

23. Kelly, M., Burke, J., Smith, M., Klar, A. \& Beach, D. (1988) EMBO J. 7, 1537-1547.

24. Kolodrubetz, D. \& Burgum, A. (1990) J. Biol. Chem. 265, 3234-3239.

25. Sinclair, A. H., Berta, P., Palmer, M. S., Hawkins, J. R., Griffiths, B. L., Smith, M. J., Foster, J. W., Fischauf, A.-M., Lovell-Badge, R. \& Goodfellow, P. N. (1990) Nature (London) 346, 240-244.

26. Bell, S. P., Learned, M., Jantzen, H.-M. \& Tjian, R. (1988) Science 241, 1192-1197.

27. Einck, L. \& Bustin, M. (1985) Exp. Cell Res. 156, 295-310.

28. Kolodrubetz, D., Haggren, W. \& Burgum, A. (1988) FEBS Lett. 238, 175-179.

29. Pikaard, C. S., McStay, B., Schultz, M. C., Bell, S. P. \& Reeder, R. H. (1989) Genes Dev. 3, 1779-1788.

30. Flaherty, K. M., DeLuca-Flaherty, C. \& McKay, D. B. (1990) Nature (London) 346, 623-628.

31. Myers, A. M., Pape, L. K. \& Tzagoloff, A. (1985) EMBO J. 4, 20872092.

32. Parisi, M. A. \& Clayton, D. A. (1991) Science 250, 965-969.

33. Parikh, V. S., Morgan, M. M., Scott, R., Clements, L. S. \& Butow, R. A. (1987) Science 235, 576-580.

34. Marczynski, G. T., Schultz, P. W. \& Jaehning, J. A. (1989) Mol. Cell. Biol. 9, 3193-3202.

35. Thoma, F., Bergman, L. W. \& Simpson, R. T. (1984) J. Mol. Biol. 177, 715-733.

36. Long, C. M., Brajkovich, C. M. \& Scott, J. F. (1985) Mol. Cell. Biol. 5, 3124-3130. 